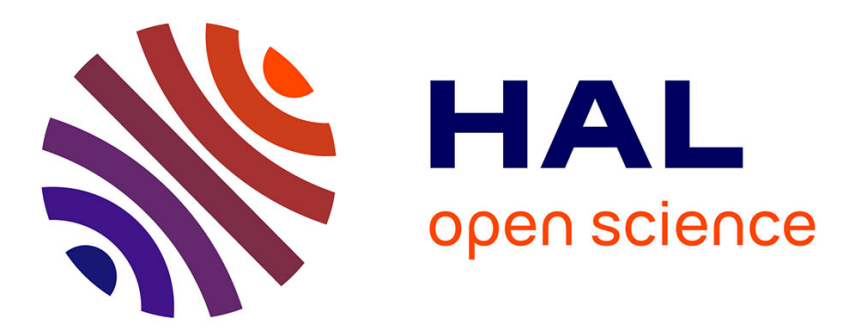

\title{
Formalizations of the Retinex model and its variants with variational principles and partial differential equations
}

Edoardo Provenzi

\section{- To cite this version:}

Edoardo Provenzi. Formalizations of the Retinex model and its variants with variational principles and partial differential equations. Journal of Electronic Imaging, 2017. hal-02480258

\section{HAL Id: hal-02480258 \\ https://hal.science/hal-02480258}

Submitted on 15 Feb 2020

HAL is a multi-disciplinary open access archive for the deposit and dissemination of scientific research documents, whether they are published or not. The documents may come from teaching and research institutions in France or abroad, or from public or private research centers.
L'archive ouverte pluridisciplinaire HAL, est destinée au dépôt et à la diffusion de documents scientifiques de niveau recherche, publiés ou non, émanant des établissements d'enseignement et de recherche français ou étrangers, des laboratoires publics ou privés. 


\title{
Formalizations of the Retinex model and its variants with variational principles and partial differential equations
}

\author{
Edoardo Provenzi \\ Université de Bordeaux, CNRS, Bordeaux INP, IMB, UMR 5251, F-33400, Talence, France
}

\begin{abstract}
Edwin H. Land and John J. McCann introduced the Retinex model as a computational theory of color vision. However, Retinex details were described rather algorithmically than mathematically. Soon after the birth of Retinex, Horn proposed a drastic simplification of the model, which, however, had the merit of being mathematically explicit and rigorous. As a consequence, at least two different interpretations of Retinex appeared in the literature almost at the same time. This generated a lot of confusion about the name Retinex that persists even nowadays. The aim of this paper is to present an overview about the different interpretations and the corresponding mathematical formalizations of Retinex in terms of variational principles and partial differential equations.
\end{abstract}

Keywords: Retinex, partial differential equations, variational principles.

provenzi.edoardo@gmail.com

\section{Introduction}

The most popular paper about the original Retinex formulation is. ${ }^{1}$ Retinex stands for 'Retina plus Cortex', which refers to the fact that the mechanisms underlying human color vision depend both on the retinal photoreceptors catches and on the cortex interpretation of these signals. The original Retinex is a computational model with the aim of finding a perceptual correlate of reflectance, called 'lightness' by Land, to be tested with psychophysical measurements.

Through a series of groundbreaking experiments, mostly performed with the famous 'Mondrian tableaux', Land and McCann proved that human perception of a surface's color is influenced by the spatial distribution of the surrounding surfaces, see Figure 1. As underlined by McCann in many papers and conference speeches, spatial locality of color perception is the central concept in the whole Retinex theory. Thus, at least in its original form, Retinex is not an algorithm for discarding illumination and recover the intrinsic reflectance of surfaces, as several authors claim in their papers even nowadays, but it is a computational model that uses the local information of the 
surround to recover color perception.

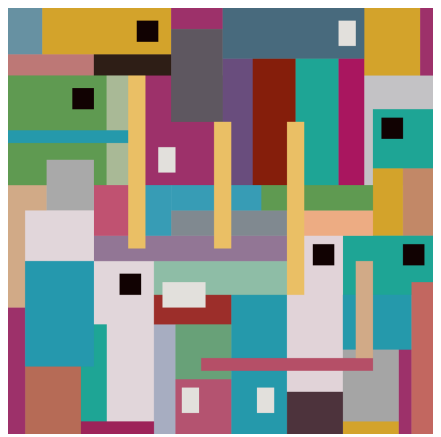

Fig 1 Digital (personal) reproduction of a typical Mondrian tableau used by Land and McCann for their color matching experiments.

In spite of their innovative and important experimental achievements, neither Land nor McCann 'carved their model into stone' through a rigorous mathematical formulation and this generated confusion, both about the role of the operations involved in the lightness computation, and in the essence of their model.

In this paper, we are not going to discuss Retinex as a vision model (see ${ }^{2}$ for more details about this), but the color enhancement algorithms for image processing that were inspired by it. In particular, we will show that two major classes of Retinex that can be found in the literature: ratio-reset Retinex and Horn's Retinex. ${ }^{3}$ We will underline how profound is the difference between these two interpretations thanks to variational principles and partial differential equations.

The variational models that will be discussed in this paper are the variational descriptions of existing models, it is thus necessary to introduce, in the following section, the basic formalization of the ratio-reset formula developed in. ${ }^{4}$ This will help us fix the ideas and the notation about many concepts and notations that will be discussed in the following sections.

It is important to keep in mind that, since the Retinex theory attempts to estimate human color sensation, pre- and post- calibrations are required to map the device responses into human re- 
sponses. Here, since we will concentrate on image processing purposes, we will not take into account the calibrations procedures.

As a final remark: this paper is about mathematical formulations of Retinex models, their similarities and differences, not about their outputs, which strongly depend on their parameters. Coherently with this, no Retinex output will be presented in this paper.

\section{Land and McCann's ratio-threshold-reset Retinex model}

As previously mentioned, the original Retinex model of Land and $\mathrm{McCann}^{1}$ is based on the assumption that the HVS operates with three retinal-cortical systems, each processing independently the low, middle and high wavelengths of the visible electromagnetic spectrum. Every independent process forms a separate image determining a quantity that they called lightness and denoted with $L$. Land and McCann found a computational way to reproduce lightness for their Mondrian tableaux by introducing spatial comparisons among intensities, calculated over paths. The comparison is performed through a multiplicative chain of ratios, subjected to these non-linear operations: Threshold mechanism: if the ratio does not differ from 1 more than a fixed threshold value, then it is set to be unitary; Reset mechanism: if the cumulated product of ratios overcomes the value 1 in a certain point of the path, then it is forced to 1 , so that the computation restarts from it. In this way, this point becomes a local white reference, so that the reset mechanism is responsible for the white-patch behavior of Retinex.

Let us now present the mathematical formalization of the ratio-threshold-reset Retinex computation described in $^{4}$ as an interpretation of. ${ }^{1}$ Other possible interpretations exist in the literatures, see e.g. ${ }^{2}$ Given a discrete digital image function with normalized range, $I: \Omega \subset \mathbb{Z}^{2} \rightarrow(0,1]$, consider a collection of $N$ oriented paths $\vec{\gamma}=\left\{\gamma_{1}, \ldots, \gamma_{N}\right\}$ composed by ordered chains of pixels 
starting in $y_{k}$ and ending in $x, k=1, \ldots, N$. Let $n_{k}$ be the number of pixels traveled by the path $\gamma_{k}$ and let $t_{k}=1, \ldots, n_{k}$ be its parameter, i.e. $\gamma_{k}:\left\{1, \ldots, n_{k}\right\} \rightarrow \Omega \subset \mathbb{R}^{2}, \gamma_{k}(1)=y_{k}$ and $\gamma_{k}\left(n_{k}\right)=x$. Write, for simplicity, two subsequent pixels of the path as $\gamma_{k}\left(t_{k}\right)=y_{t_{k}}$ and $\gamma_{k}\left(t_{k}+1\right)=y_{t_{k}+1}$, for $t_{k}=1, \ldots, n_{k}-1$. Consider, in every fixed chromatic channel $c \in\{R, G, B\}$, their intensities $I\left(y_{t_{k}}\right), I\left(y_{t_{k}+1}\right)$ and then compute the ratio $R_{t_{k}}=\frac{I\left(y_{t_{k}+1}\right)}{I\left(y_{t_{k}}\right)}$ with the initial condition $R_{0}=1$.

With this notation in mind, the value of lightness provided by the ratio-threshold-reset Retinex algorithm for a generic pixel $x \in \Omega$, in every fixed chromatic channel $c$ (that we avoid specifying for the same of a clearer notation), is given by:

$$
L_{\varepsilon, \vec{\gamma}}(x)=\frac{1}{N} \sum_{k=1}^{N} \prod_{t_{k}=1}^{n_{k}-1} \delta_{k}\left(R_{t_{k}}\right)
$$

where $\delta_{k}: \mathbb{R}^{+} \rightarrow \mathbb{R}^{+}, k=1, \ldots, N$, are functions defined in this way: $\delta_{k}\left(R_{0}\right)=1$ and, for $t_{k}=1, \ldots, n_{k}-1$

$$
\delta_{k}\left(R_{t_{k}}\right)= \begin{cases}R_{t_{k}} & \text { if } 0<R_{t_{k}} \leq 1-\varepsilon \\ 1 & \text { if } 1-\varepsilon<R_{t_{k}}<1+\varepsilon \\ R_{t_{k}} & \text { if } 1+\varepsilon \leq R_{t_{k}} \leq \frac{1+\varepsilon}{\prod_{m_{k}=0}^{t_{k}-1} \delta_{k}\left(R_{m_{k}}\right)} \\ \frac{1}{\prod_{m_{k}=0}^{t_{k}-1} \delta_{k}\left(R_{m_{k}}\right)} & \text { if } R_{t_{k}}>\frac{1+\varepsilon}{\prod_{m_{k}=0}^{t_{k}-1} \delta_{k}\left(R_{m_{k}}\right)}\end{cases}
$$

being $\varepsilon>0$ a fixed threshold. The second option is the mathematical implementation of the threshold mechanism while the fourth implements the reset mechanism (and so the white patch behavior) of the algorithm. 
It is useful to write the contribution of the single path $\gamma_{k}$ to $L_{\varepsilon, \vec{\gamma}}(x)$ as:

$$
L_{\varepsilon, \gamma_{k}}(x)=\prod_{t_{k}=1}^{n_{k}-1} \delta_{k}\left(R_{t_{k}}\right)
$$

so that formula (1) reduces simply to the average of these contributions, i.e. $L_{\varepsilon, \vec{\gamma}}(x)=\frac{1}{N} \sum_{k=1}^{N} L_{\varepsilon, \gamma_{k}}(x)$.

Before passing to the analysis of the limit $\varepsilon \rightarrow 0$, it is worthwhile specifying two issues. Firstly, an independent processing of the three chromatic channels is a correct assumption only for the very first stages of human vision. In fact, as soon as the visual signal arrives to ganglion cells, the output signals coming from the photoreceptors start to intertwine..$^{5-7}$ Secondly, the ratio-threshold-reset algorithm described above is implemented in the so-called Milano Retinex algorithms ${ }^{2}$ to perform color enhancement in digital images through a spatial color processing inspired by the Retinex principles.

\subsection{The limit behavior $\varepsilon \rightarrow 0$}

The analytical formula to describe the ratio-threshold-reset Retinex algorithm just introduced allowed making predictions about the model. As explained in, ${ }^{4}$ this can be done if the threshold mechanism is disregarded, or, equivalently, by considering the case $\varepsilon \rightarrow 0$.

As $\varepsilon \rightarrow 0$, the functions $\delta_{k}$ become much simpler:

$$
\delta_{k}\left(R_{t_{k}}\right)= \begin{cases}R_{t_{k}} & \text { if } 0<R_{t_{k}} \prod_{m_{k}=0}^{t_{k}-1} \delta_{k}\left(R_{m_{k}}\right) \leq 1 \\ \frac{1}{\prod_{m_{k}=0}^{t_{k}-1} \delta_{k}\left(R_{m_{k}}\right)} & \text { if } R_{t_{k}} \prod_{m_{k}=0}^{t_{k}-1} \delta_{k}\left(R_{m_{k}}\right)>1\end{cases}
$$

hence, when $\varepsilon \rightarrow 0, \delta_{k}$ behaves either as the identity or the reset function. 
$\mathrm{In}^{4}$ it was proven that this implies the following formula:

$$
L_{0, \vec{\gamma}}(x)=\frac{1}{N} \sum_{k=1}^{N} \frac{I(x)}{I\left(y_{H_{k}}\right)},
$$

where $y_{H_{k}}$ is the pixel with highest intensity traveled by $\gamma_{k}$. From now on, we will refer to formula (5) as describing the 'ratio-reset Retinex algorithm'.

Notice that he presence of paths makes the ratio-reset Retinex a local algorithm, where locality is intrinsically represented by the geometry of paths used. However, when $n_{k} \rightarrow|\Omega|$, the ratioreset Retinex loses its local properties and reduces, see, ${ }^{4}$ to the global diagonal von Kries model ${ }^{8}$ On the other hand, $n_{k}$ and $N$ contribute to the creation of noise when their value is small.

Finally, it is important to underline that, since intensity values are normalized, $0<I\left(y_{H_{k}}\right) \leq 1$ for every $k=1, \ldots, N$ and then $\sum_{k=1}^{N} \frac{1}{I\left(y_{H_{k}}\right)} \geq N$. It follows that $L(x) \geq I(x)$ for every pixel $i$ and this proves that an image filtered with the ratio-reset Retinex is always brighter or equal to the original one. This shows an important limitation of this algorithm: an over-exposed picture can only be worsened by the application of the ratio-reset Retinex used as a color corrector.

\subsection{From paths to pixel sprays: RSR and related algorithms}

The information obtained thanks to the mathematical formulation of Retinex has important consequences on the structure of $\mathcal{P}_{x}(\Omega)$, the set of paths embedded in the image domain $\Omega$ and ending in the point $x$. After formula (5), on this set it is natural to define the following equivalence relation: given $\gamma, \eta \in \mathcal{P}_{x}(\Omega)$

$$
\gamma \sim \eta \quad \Leftrightarrow \quad \max _{y \in \gamma^{*}}\{I(y)\}=\max _{y \in \eta^{*}}\{I(y)\}
$$


where $\gamma^{*}$ and $\eta^{*}$ are the co-domains of the paths, i.e. the collections of pixels traveled by $\gamma$ and $\eta$, respectively.

Paths belonging to different equivalence classes give different contributions to the lightness computation, while every path in a given equivalence class gives rise to the same value of $L_{0, \gamma_{k}}(x)$. It follows immediately that $\mathcal{P}_{x}(\Omega)$ contains redundant paths and that the correct set of paths to consider is given by the quotient set $\mathcal{P}_{x}(\Omega) / \sim$, whose elements are the equivalence classes of paths with respect to the equivalence relation defined in (6).

In each equivalence class one can choose a single representative path to compute $L_{0, \gamma_{k}}(x)$, in particular, the most efficient one is the two-points path whose co-domain is simply given by $\left\{y_{H_{k}}, x\right\}$. Moreover, by a mathematical point of view, paths are topological manifolds of dimension 1 embedded in the image, which is a topological manifold of dimension 2, so paths do not really scan local neighborhoods of a pixel, rather particular directions in these neighborhoods. This directional extraction of information can lead to halos or artifacts in the filtered image, see e.g. ${ }^{9}$

These considerations led the authors of ${ }^{10}$ to consider 2-dimensional objects such as areas instead of 1-dimensional paths to analyze image locality for an efficient color correction. Roughly speaking, their idea is to implement spatial locality by selecting a fraction of pixels from these areas with a density sample that changes according to a given function of their distance with respect to the target pixel $x$. Each function generates a different kind of pixel selection around $x$, leading to different kind of 'sprays', as the one depicted in Figure 2, each of which shows different local filtering properties. The new implementation of the ratio-reset Retinex that follows this idea is called RSR for 'Random Sprays Retinex'.

In RSR the role of a path $\gamma_{k}$ traveling $n_{k}$ pixels and ending in the target $x$ is played by $S_{k}(x)$, a spray with $n_{k}$ pixels centered in $x$. Actually, once the number of points per spray is chosen, there 


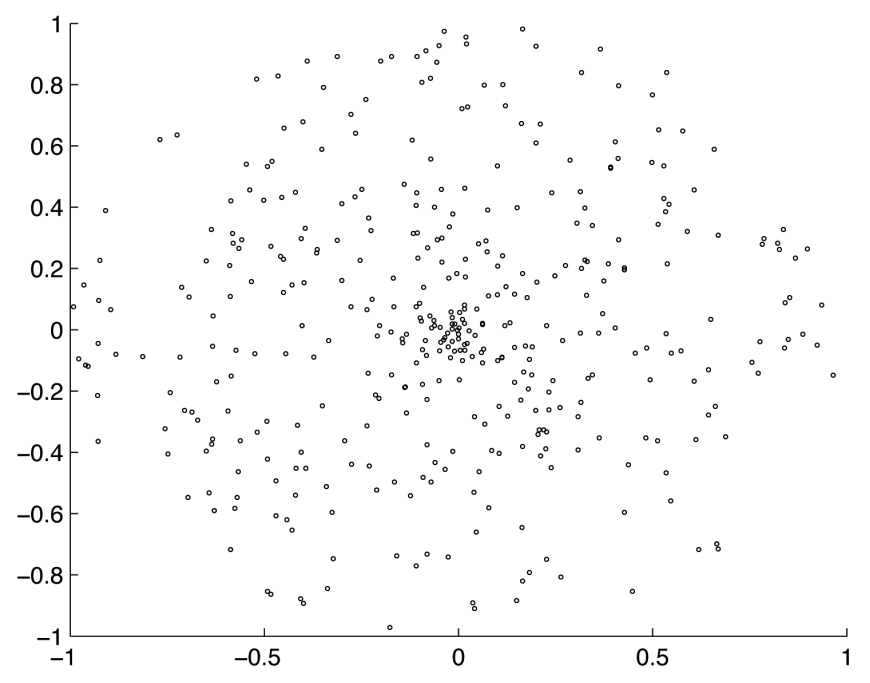

Fig 2 An example of (normalized) random spray. Normalization refers to the fact that the horizontal and vertical axes have been normalized between 0 and 1 .

is no need to vary it with $k$, hence, from now on, we will write $n$ instead of $n_{k}$ to denote the number of pixels per spray. The ratio-reset operation along a path is substituted by the search of the pixel with highest intensity in the whole spray. The functional expression of formula (5) to compute the lightness remains exactly the same in both algorithms, so the ratio-reset Retinex and RSR share the same intrinsic properties.

$\operatorname{In}^{11,12}$ two techniques have been proposed to reduce noise generation also decreasing the computational time of RSR.

Let us start by describing the strategy described in $^{11}$ and called 'Light Random Sprays Retinex' (LRSR) devised to avoid noise formation when a small value of $n$ and/or $N$ is used. Consider an arbitrary input image $I$ and apply RSR to it, obtaining the image $L$, the ratio $C=\frac{I}{L}$ is called intensity change image. In LRSR, the noise is reduced through a convolution with a kernel function $k$. This can be done after the computation of $C$, obtaining $C_{k}^{\prime}=(C * k)(x), \forall x \in \Omega$, or before it, i.e. applying the blurring on $I$ and $L$, obtaining $C_{k}^{\prime \prime}(x)=\frac{(I * k)(x)}{(L * k)(x)}, \forall x \in \Omega$. By combining the two 
approaches, i.e filtering before and after calculation with two (possibly identical) kernels $k_{1}$ and $k_{2}$, respectively, one can define the new intensity change matrix: $C_{k_{1}, k_{2}}^{*}(x)=\left(C_{k_{1}}^{\prime \prime}(x) * k_{2}\right)(x)$.

The output image $O$ of LRSR is calculated via the formula:

$$
O(x)=\frac{I(x)}{C_{k_{1}, k_{2}}^{*}(x)} \quad \forall x \in \Omega,
$$

the size of kernels $k_{1}$ and $k_{2}$ used in ${ }^{11} 25 \times 25$.

In, ${ }^{12}$ the same authors attacked the problem of computational complexity reduction with a technique called 'Smart Light Random Memory Sprays Retinex' (SLRMSR). The basic concept behind SLRMSR is that of 'spray memory', which consists in creating a single spray that will be gradually modified while browsing the image by modifying just one pixel of $S(x)$ each time we move from one pixel to another, by randomly selecting a pixel that lies in the neighborhood of the last pixel. By iterating this procedure, the original spray is gradually and smoothly modified. Thanks to this idea, the computational complexity passes from $\mathcal{O}(n N|\Omega|)$ to $\mathcal{O}(n|\Omega|)$ because now one spray is used. In, ${ }^{13}$ these techniques have been extended from RSR to RACE.

$\mathrm{In}^{14}$ the spray technique was used to fuse RSR with ACE, ${ }^{15}$ another perceptually-inspired color correction algorithm that makes use of the gray-world hypothesis. ${ }^{16}$ The hybrid algorithm is called RACE and it is able to color correct both under and over exposed images.

A more recent proposal to fuse white-patch (WP) and gray-world (GW) features in a single algorithm is that presented in ${ }^{17}$ and called STRESS for Spatio-Temporal Retinex-like Envelope with Stochastic Sampling. As Retinex, STRESS computes, for each pixel, the local white reference, but also the black reference in each chromatic channel. This is done through calculating the maximum and minimum envelope functions, denoted as $E_{\max }(x)$ and $E_{\min }(x)$, respectively. 
The computational steps needed to get the envelope functions are the following: fix a pixel $x \in$ $\Omega$ and $N$ random sprays ${ }^{10} S_{k}(x), k=1, \ldots, N$, centered in $x$, then compute $M_{k}=\max _{x \in S_{k}(x)} I(x)$, $m_{k}=\min _{x \in S_{k}(x)} I(x)$ and the following objects:

$$
r_{k}(x)=M_{k}(x)-m_{k}(x), \quad v_{k}(x)= \begin{cases}\frac{1}{2} & \text { if } M_{k}(x)=m_{k}(x) \\ \frac{I(x)-m_{k}(x)}{M_{k}(x)-m_{k}(x)} & \text { otherwise }\end{cases}
$$

thus, essentially, the value of $v_{k}(x)$ is either the middle gray if $x$ lies in a homogeneous area, or it is the linearly stretched value of $I(x)$ with respect to the interval $\left[m_{k}(x), M_{k}(x)\right]$. By denoting with $\bar{r}(x)$ and $\bar{v}(x)$ the average values of $r_{k}(x)$ and $v_{k}(x)$ over the $N$ sprays, i.e.

$$
\bar{r}(x)=\frac{1}{N} \sum_{k=1}^{N} r_{k}(x), \bar{v}(x)=\frac{1}{N} \sum_{k=1}^{N} v_{k}(x),
$$

the authors can finally define the two envelope functions $E_{\min }(x)$ as follows:

$$
E_{\min }(x)=I(x)-\bar{v}(x) \bar{r}(x), \quad E_{\max }(x)=I(x)+(1-\bar{v}(x) \bar{r}(x))=E_{\min }+\bar{r}(x) .
$$

The final output of STRESS is the stretched value of $I(x)$ on the interval $\left[E_{\min }, E_{\max }\right]$, namely:

$$
\operatorname{STRESS}(I(x))=\frac{I(x)-E_{\min }}{E_{\max }-E_{\min }} .
$$

STRESS, as RACE, is capable of handling both under and over exposed images, but it is affected by the same noise problems of RSR. In section 3 we will see a variational extension of the technique used to compute the envelope functions based on the total variation. This will allow avoiding the 
noise problems related to the random spray technique.

Finally, let us mention some related works. In, ${ }^{18}$ the RSR sampling technique has been studied from a probabilistic point of view, resulting in the algorithm QBRIX, in, ${ }^{19}$ further comparisons among Retinex models are discussed, $\mathrm{in}^{20}$ a noise-free, exact mapping of RSR into a probabilistic framework is presented, in ${ }^{21}$ fuzzy variants of RSR are taken into account and, finally, in ${ }^{22}$ a random spatial sampling method halfway through path-based and 2D-based is discussed.

\section{A variational framework for the ratio-reset Retinex}

The similarities between the ACE formula ${ }^{15}$ and the gradient descent equations for histogram equalization obtained in, ${ }^{23}$ led to the discovery of a variational interpretation of ACE in the paper. $^{24}$ The framework were further extended $i^{25}$ and, finally, $i^{26}$ a variational framework for (an anti-symmetric version of the) ratio-reset Retinex has been discussed. In order to understand how this is possible, let us come back to the lightness formula (5). An algorithmic analysis of these models has been provided in. ${ }^{27}$ Interestingly, this variational setting can be also extended to consider cognitive phenomena, as it has been shown in. ${ }^{28}$

Land proposed a further Retinex mechanism, the scaling, ${ }^{29}$ implemented via a strictly increasing compressive (concave) function $f:(0,1] \rightarrow(0,1]$ such that $f(r) \geq r$ for all $r \in(0,1]$ applied to the ratio $r=\frac{I(x)}{I\left(y_{H_{k}}\right)}$, so that the Retinex lightness formula becomes:

$$
L_{0, \vec{\gamma}, f}(x)=\frac{1}{N} \sum_{k=1}^{N} f\left(\frac{I(x)}{I\left(y_{H_{k}}\right)}\right) .
$$

The reset mechanism of Retinex and the scaling operation can be merged: in fact, we can extend 
$f$ to $(0,+\infty)$ preserving its continuity by defining

$$
\hat{f}(r)= \begin{cases}f(r) & \text { if } r \in(0,1] \\ 1 & \text { if } r \in[1,+\infty) .\end{cases}
$$

It is clear that applying this new scaling function $\hat{f}$ to the ratios $I(x) / I(y)$, with $x$ fixed and $y$ that varies in $\Omega$, jointly implements the scaling and the reset mechanism.

Now we have all the elements to introduce the continuous version of the Retinex algorithm presented in $^{26}$ under the name 'Kernel-Based Retinex', or KBR for short. Given $x \in \Omega$, let $Y_{w, x}$ be the random variable modeling the selection of a pixel in the neighborhood of $x$ according to the density $w(x, y)$.

The output $L_{w}^{\mathrm{KBR}}(x)$ of the KBR algorithm at the pixel $x$ is defined as the conditional expectation of $\hat{f}\left(\frac{I(x)}{I\left(Y_{w, x}\right)}\right)$ with respect to the distribution $w$ of pixels around $x$, i.e.

$$
L_{w}^{\mathrm{KBR}}(x)=\mathbb{E}_{Y_{w, x}}\left[\hat{f}\left(\frac{I(x)}{I\left(Y_{w, x}\right)}\right)\right]
$$

This formula is used independently for each color channel and can be written more explicitly as

$$
L_{w}^{\mathrm{KBR}}(x)=\sum_{\{y \in \Omega: I(y) \geq I(x)\}} w(x, y) f\left(\frac{I(x)}{I(y)}\right)+\sum_{\{y \in \Omega: I(y)<I(x)\}} w(x, y)
$$

All the basic properties of the ratio-reset Retinex are faithfully implemented in (12): KBR is founded on the propagation of a two-pixel ratio comparison between the fixed target $x$ and the generic pixel $y$ that runs across the image; these comparisons are then subjected to the reset and scaling performed by $\hat{f}$ and, finally, locally averaged with weight $w$, in order to produce the value 
of $L_{w}^{\mathrm{KBR}}(x)$.

To study the action of KBR of pixel intensities, it is useful to rewrite (12) introducing the functions

$$
\operatorname{sign}^{+}(\xi):=\left\{\begin{array}{rl}
1 & \text { if } \xi>0, \\
\frac{1}{2} & \text { if } \xi=0, \\
0 & \text { if } \xi<0,
\end{array} \quad \operatorname{sign}^{-}(\xi)=1-\operatorname{sign}^{+}(\xi),\right.
$$

so that eq. (12) can be re-written as

$$
L_{w}^{\mathrm{KBR}}(x)=\sum_{y \in \Omega} w(x, y) f\left(\frac{I(x)}{I(y)}\right) \operatorname{sign}^{+}(I(y)-I(x))+\sum_{y \in \Omega} w(x, y) \operatorname{sign}^{-}(I(y)-I(x)) .
$$

Thanks to eq. (13) we can verify that KBR always increases brightness as the ratio-reset Retinex implementation. In fact, since $f(r) \geq r$ for all $r \in(0,1]$, then $f\left(\frac{I(x)}{I(y)}\right) \geq \frac{I(x)}{I(y)} \geq I(x)$, so

$$
L_{w}^{\mathrm{KBR}}(x) \geq \sum_{y \in \Omega} w(x, y) I(x) \operatorname{sign}^{+}(I(y)-I(x))+\sum_{y \in \Omega} w(x, y) \operatorname{sign}^{-}(I(y)-I(x))
$$

moreover, being $I(x) \leq 1$, we can write

$$
\begin{aligned}
L_{w}^{\mathrm{KBR}}(x) & \geq \sum_{y \in \Omega} w(x, y) I(x) \operatorname{sign}^{+}(I(y)-I(x))+\sum_{y \in \Omega} w(x, y) I(x) \operatorname{sign}^{-}(I(y)-I(x)) \\
& =I(x) \sum_{y \in \Omega} w(x, y)\left[\operatorname{sign}^{+}(I(y)-I(x))+\operatorname{sign}^{-}(I(y)-I(x))\right] \\
& =I(x) \sum_{y \in \Omega} w(x, y)=I(x)
\end{aligned}
$$

having used the fact that the kernel is normalized. As in the ratio-reset formulation, this property implies that over-exposed pictures could not be enhanced with Retinex unless we use a post- 
processing stage and that further iterations of Retinex keep on increasing the intensity until a white image is reached.

This equation of KBR does not correspond to the minimization of an energy functional. However, let us consider the sum of the function $f\left(\frac{I(x)}{I(y)}\right) \operatorname{sign}^{+}(I(y)-I(x))$ and of its the antisymmetrized version on the region $\{x \in \Omega: I(y) \leq I(x)\}$, i.e.

$$
\begin{aligned}
L_{w}^{\mathrm{aKBR}}(x) & =\sum_{y \in \Omega} w(x, y) f\left(\frac{I(x)}{I(y)}\right) \operatorname{sign}^{+}(I(y)-I(x)) \\
& -\sum_{y \in \Omega} w(x, y) f\left(\frac{I(y)}{I(x)}\right) \operatorname{sign}^{-}(I(y)-I(x))
\end{aligned}
$$

where aKBR stands for anti-symmetrized KBR.

$\mathrm{In}^{26}$ it was proven that the right-hand side of the previous equation can be interpreted as the minimization of the energy functional given by:

$$
C_{w}^{f}(I)=\sum_{x \in \Omega} \sum_{y \in \Omega} w(x, y) f\left(\frac{\min (I(x), I(y))}{\max (I(x), I(y))}\right) .
$$

Minimizing $C_{w}^{f}(I)$ corresponds to maximizing the contrast in a local (due to the presence of the weight $w$ ) and non linear way (due to the ratio and to the presence of $f$ ). In fact, since the functional $C_{w}^{f}(I)$ is defined via a quotient, its argmin will balance the minimization of the numerator and maximization of the denominator, thus the minimal and the maximal intensity will be spread apart and this will generate a contrast enhancement. This explained in a quantitative and qualitative way how and why the somewhat involved ratio-reset mechanism of Retinex allows for a unilateral contrast enhancement, always directed towards the highest intensity.

KBR, ACE, RACE and STRESS corrected this unilateral behavior. $\operatorname{In}^{30}$ the spatially-based 
variational framework was translated into a wavelet-based setting.

\section{Retinex: a 'melody' that everyone plays differently}

In image processing it is hard to find a model whose name has been interpreted in so many different ways as 'Retinex'. In this section, we present a synthetic description of the evolution of the Retinex interpretation.

Path-wise Retinex share a local WP nature and mostly differ from each other by the path geometry used to explore spatial locality: Land and McCann used piecewise linear paths in. ${ }^{1} \operatorname{In}^{31,32}$ and $^{33}$ those paths were substituted by double spirals, Brownian paths and traces of a specialized swarm of termites, respectively.

Center/surround Retinex are local GW algorithms originated from, ${ }^{34}$ where Land noticed that he could reproduce Mach bands originated by a spinning white square on a black background by using a different Retinex formulation. Precisely, for every image point, the intensity of the center $x$ is replaced by the ratio between $I(x)$ and the average value of the surround, sampled with a density that decays as the inverse of the square distance from the center. Writing with $L^{\mathrm{CS}}$ this 'center/surround lightness', we have: $L^{\mathrm{CS}}(x)=I(x) /<\{I(y), y \in$ Surround $\}>$, where $<\cdot>$ represents the average operator. Comparing this last formula with (5), it can be seen that there is a fundamental difference between this formulation and the ratio-reset one: there the ratio is performed over the pixel with highest intensity, while in this formulation it is implemented over the mean value of the surround. In practice, this last formulation can be seen as a gray-world method to remove the illuminant component of the image. ${ }^{16}$

In 1997, Johbson, Rahman and Woodell ${ }^{35}$ re-elaborated Land's idea presented in: ${ }^{34}$ they worked with logarithmic data, approximating the average of the surround by convolving the image func- 
tion $I$ with a normalized kernel function $F$, usually a Gaussian. If we use again, for simplicity, the symbol $L^{\mathrm{CS}}$, we can write this model as follows: $L^{\mathrm{CS}}(x)=\log (I(x))-\log ((F * I)(x)), \forall x \in \Omega$. Multilevel Retinex algorithms were pioneered by Frankle and McCann in ${ }^{9}$ and further refined in. ${ }^{36}$ In these works a multilevel version of the ratio-reset local WP Retinex is presented, the authors abandon paths and consider a computation that takes into account all pixels. The input image is progressively sub-sampled averaging a number of pixel that grows as increasing powers of 2. On each sub-sample level a ratio-reset computation (without threshold) is operated a certain number of times, from the coarser sub-sample level to the finest one. Because of the sub-sampling, as we go far from the target pixel, we do not consider actual pixel values, but average values of macroareas of increasing size. A rigorous mathematical formulation of these multilevel algorithms is still lacking.

Based on this idea, Marini, Rizzi and De Carli ${ }^{32}$ constructed a local WP multilevel version of Brownian path Retinex that reduced the amount of noise in the output images. A different multilevel proposal has been pointed out by Johbson, Rahman and Woodell in: ${ }^{37}$ they introduced a certain number $S$ of scales where performing the convolutions with normalized Gaussian functions $F_{s}, s=1, \ldots, S$. Each scale is associated to a suitable weight $w_{s}$, which gives more importance to finer scales than to coarser ones.

Finally there are WP Retinex versions based on solving a Poisson equation. They rely on a work of Horn, ${ }^{3}$ in which he remarkably pointed out, for the first time, the need for a spatially isotropic two-dimensional version of Retinex. Horn considered, as Land, only Mondrian tableaux illuminated by a smoothly varying light. However, differently from Land, he explicitly tackled the ill-posed problem of inverting the equation $I_{c}(x)=S_{c}(x) L_{c}(x), c \in\{R, G, B\}$, with respect to $S_{c}(x)$, the reflectance of the point $x$, knowing only the image intensity $I_{c}(x)$ and not the 
illumination $L_{c}(x)$. If we pass to $\log$ arithmic values, i.e. $\log I_{c}(x)=\log S_{c}(x)+\log L_{c}$ or, equivalently, $\log S_{c}(x)=\log I_{c}(x)-\log L_{c}$ and we apply a differential operator $D$ to both sides, then $D\left(\log L_{c}(x)\right)$ will be small but finite everywhere, while $D\left(\log S_{c}(x)\right)$ will be different from zero only if $x$ is close to sharp edges.

If we apply a threshold operator $\delta_{T}$ defined as follows:

$$
\delta_{T}(s)= \begin{cases}s & \text { if }|s|>T \\ 0 & \text { elsewhere }\end{cases}
$$

for all $s \in \mathbb{R}$ and if the threshold $T>0$ is small enough, then we obtain $D\left(\log S_{c}(x)\right)=$ $\delta_{T}\left(D\left(\log I_{c}(x)\right)\right)$. Horn insisted on the choice of the Laplacian for $D$ instead of the gradient, arguing that first order derivatives are one-dimensional, while the second order derivatives involved in the Laplacian are isotropic and thus more suited for the topology of an image. By substituting $D$ with the Laplacian operator $\Delta$, the last formula becomes a Poisson equation:

$$
\Delta\left(\log S_{c}(x)\right)=\delta_{T}\left(\Delta\left(\log I_{c}(x)\right)\right)
$$

whose solution allows to recover the logarithmic reflectance $\log S_{c}(x)$. It is clear that Horn's method is based on quite restrictive hypotheses: smoothness of illumination (violated by scenes with deep shadows, for instance) and a Mondrian-like world (violated each time edges are not sharp). 


\section{Mathematical formalizations of Horn's interpretation}

Besides the variational framework described in section 3 , in the literature there exist alternative variational models of Retinex-like algorithms and also formalizations based on partial differential equations (PDE). The aim of this section is not to give an exhaustive list, rather to discuss the main features of the most famous alternative mathematical formalizations of Retinex-like algorithms present in the literature.

The first authors to embed a Retinex-like algorithm in a variational framework were Kimmel and colleagues in. ${ }^{38}$ They did not considered the ratio-threshold-reset Retinex, but Horn's interpretation. In fact, they started from the logarithmic equation $\log I_{c}(x)=\log S_{c}(x)+\log L_{c}(x)$, $c \in\{R, G, B\}$ and tried to solve it with respect to $\log L_{c}(x)$ by imposing the hypothesis of smoothness on the illuminant part of the logarithmic image. Once obtained an estimation of the illumination, they could infer the reflectance information $S_{c}(x)$. This one then undergoes suitable transformations and gives an illuminant-invariant version of the original image.

It is important to underline a fundamental difference between this variational technique and the one presented in the previous sections: here contrast enhancement of the original image $\log I_{c}(x)$ is obtained by decreasing the contrast of the illuminant image $\log L_{c}(x)$. In fact, $\log I_{c}(x)$ is measured by the camera and so it is a fixed data, $\log L_{c}(x)$ is estimated by using a smoothness prior, thus the estimated reflectance $\log S_{c}(x)=\log I_{c}(x)-\log L_{c}(x)$, or $S_{c}(x)=I_{c}(x) / L_{c}(x)$ is forced to have a stronger contrast than the original image data. Instead, the variational principles previously discussed act directly on the contrast of the original image, without taking into account the separation between reflectance and illuminant and related approximations and priors.

Avoiding the subscript $c$, the functional proposed in, ${ }^{38}$ with the notations of this paper, can be 
expressed as follows:

$$
E_{\alpha, \beta}(\log L)=\sum_{x \in \Omega}\left[|\nabla \log L(x)|^{2}+\alpha(\log L(x)-\log I(x))^{2}+\beta|\nabla(\log L(x)-\log I(x))|^{2}\right]
$$

with the constraints $\log L(x) \geq \log I(x)$, because the reflectance $S(x)$ is always between 0 and 1 , and the boundary condition $\langle\nabla \log L, \vec{n}\rangle=0$ on $\partial \Omega$, i.e. $\log L$ orthogonal to the normal $\vec{n}$ to the boundary $\partial \Omega$ of $\Omega$.

The first term of the functional forces spatial smoothness on the illumination $L$. The authors chose that particular analytical form because the Euler-Lagrange equation associated to $\sum_{x \in \Omega}|\nabla \log L(x)|^{2}$ is the Laplace PDE $\Delta \log L=0$, whose steepest descent solution is equivalent to a Gaussian smoothing. The second penalty term forces a proximity between $\log L$ and $\log I$, so that their difference $\log S$, the logarithmic reflectance, tends to 0 , i.e. the reflectance $R$ tends to 1 , or white. The authors declare that the principal objective of this term is to regularize the problem, so that it is better conditioned in view of a numerical solution and they set the constant $\alpha$ to a very small value not to force too much $\log L$ towards $\log I$. The third term represents a Bayesian penalty, which forces reflectance gradients to be smooth. The authors declared to have introduced it to force $R$ to be visually pleasing, without abrupt variations.

Morel, Petro and Sbert ${ }^{39}$ analyzed the Retinex model ${ }^{40}$ without the reset mechanism. They showed that, if the Retinex paths are interpreted as symmetric random walks, then Retinex is equivalent to the following Neumann problem for a Poisson equation:

$$
\begin{cases}-\Delta L(x)=F(x) & x \in \Omega \\ \frac{\partial L(x)}{\partial \vec{n}}=0 & x \in \partial \Omega\end{cases}
$$

where $F$ is a suitable scalar field, see ${ }^{39}$ page 2830. 
Let us now consider the algorithm STRESS. We recall that the basic information needed by STRESS is given by the two envelope functions $E_{\min }$ and $E_{\max }$ which, in the original formulation, are computed through the same random spray technique of RSR. ${ }^{10}$ To avoid the typical noise problems related to this technique, in ${ }^{41}$ the authors proposed to compute the envelope functions via the minimization of a functional based on the total variation, instead of using the random spray technique. For this reason the corresponding algorithm is called STRETV and corresponds to the minimization of the following functional for $E$ (in this case $E$ denotes the envelope and not the energy functional):

$$
\sum_{x \in \Omega}\left[|\nabla E(x)|+\frac{\lambda}{2}|E(x)-I(x)|^{2}\right]
$$

subjected to $E(x) \geq I(x)$ to compute $E_{\max }$ and to $E(x) \leq I(x)$ for $E_{\min }$.

The minimization of the first (total variation) term, assures the spatial smoothness of the envelope functions, the second term is a fidelity term used not to depart too much from the original image values. The authors declare that the coefficient $\lambda$ must be $\ll 1$ for good results. The authors do not specify if they consider a spatial kernel to localize their computation or not.

The last variational formalization that we discuss here is that presented $\mathrm{in}^{42}$ relative to the termite Retinex. ${ }^{33,43}$ Here an energy functional is taken into account to determine the geometry of the paths used by Retinex. Fixed a pixel $x \in \Omega$, the authors search for the path $\gamma:[0,1] \rightarrow \Omega$, $\gamma(0)=x$, the minimizes the energy functional defined by:

$$
E(\gamma)=\int_{0}^{1}\left[\frac{1}{1+\left(D^{2}-\|x-\gamma(s)\|^{2}\right)\|\nabla I(\gamma(s))\|^{2}}+\theta(\gamma(s))\right] d s
$$

where $D$ is the diagonal of $\Omega$ and 1 is introduced to avoid singularities in the case the denominator 
is 0 . The paths that minimize $E(\gamma)$ are those which balance the fact to remain as close to $x$ as possible and, simultaneously, to explore image areas with high values of the gradient. Both features maximize the denominator of the first term. If $x$ lies in an area with a high density of edges, $\gamma$ will not go too far from $x$, instead, if $x$ lies in a rather homogeneous area, $\gamma$ will be forced to explore the image points far away from $x$ to find the important edge information. $\theta(\gamma(s))$, the so-called 'poison term', is set to zero at the beginning, and it increases each time a path has been traveled, to prevent from exploring the same image area all the time. Once a set of $N$ path has been selected, the intensity $I(x)$ of the pixel $x$ in each separate chromatic channel is modified with the Retinex formula (5).

\section{Conclusions}

In the past fifteen years, variational methods have been used to formalize color correction algorithms. This permitted to point out similarities and differences among several models that were difficult to detect just looking at their direct equations. In this paper we have described, in a selfcontained way, both the direct and the variational versions of several color enhancement algorithms inspired by the seminal Retinex theory of color vision. A particular emphasis has been put in highlighting the very different variational formulations of the original Retinex of Land and McCann and those referring to Horn's interpretation, which are often misleadingly mixed in the literature. When the variational formulation of the ratio-reset Retinex is compared with Horn's a clear difference is underlined, both in the functional terms and in the argument of the variational energies. A more complete treatise about this subject can be found in. ${ }^{44}$ 
References

1 E. Land and J. McCann, "Lightness and Retinex theory," Journal of the Optical Society of America 61, 1-11 (1971).

2 J. McCann and A. Rizzi, The art and science of HDR imaging, vol. 26, John Wiley \& Sons (2011).

3 B. Horn, "Determining lightness from an image," Computer Graph. and Im. Proc. 3, 277-299 (1974).

4 E. Provenzi, L. De Carli, A. Rizzi, et al., "Mathematical definition and analysis of the retinex algorithm," Journal of the Optical Society of America A 22, 2613-2621 (2005).

5 G. Buchsbaum and A. Gottschalk, “Trichromacy, opponent colours coding and optimum colour information transmission in the retina," Proc. Royal Society of London B 220, 89113 (1983).

6 D. Ruderman, T. Cronin, and C. Chiao, "Statistics of cone responses to natural images: implications for visual coding," J. Opt. Soc. Am. A 15, 2036-2045 (1998).

7 E. Provenzi, J. Delon, Y. Gousseau, et al., "On the second order spatiochromatic structure of natural images," Vision research 120, 22-38 (2016).

8 J. von Kries, “Chromatic adaptation," Festschrift der Albrecht-Ludwigs-Universität, 145-158 (1902).

9 J. Frankle and J. J. McCann, "Method and apparatus for lightness imaging." United States Patent, US 4,348,336 (1983).

10 E. Provenzi, M. Fierro, A. Rizzi, et al., "Random spray retinex: A new retinex implementa- 
tion to investigate the local properties of the model," IEEE Transactions on Image Processing 16, 162-171 (2007).

11 N. Banić and S. Lončarić, "Light random sprays retinex: Exploiting the noisy illumination estimation,” IEEE Signal Processing Letters 20, 1240-1243 (2013).

12 N. Banić and S. Lončarić, "Smart light random memory sprays retinex: a fast retinex implementation for high-quality brightness adjustment and color correction," JOSA A 32(11), 2136-2147 (2015).

13 J. Chauvin and E. Provenzi, "SLMRACE: a noise-free race implementation with reduced computational time," Journal of Electronic Imaging 26(3), 031202-031202 (2017).

14 E. Provenzi, C. Gatta, M. Fierro, et al., "Spatially variant white patch and gray world method for color image enhancement driven by local contrast," IEEE Trans. on Pattern Analysis and Machine Intelligence 30, 1757-1770 (2008).

15 A. Rizzi, C. Gatta, and D. Marini, "A new algorithm for unsupervised global and local color correction," Pattern Recognition Letters 24, 1663-1677 (2003).

16 G. Buchsbaum, "A spatial processor model for object colour perception," Journal of the Franklin Institute 310, 337-350 (1980).

$17 \varnothing$. Kolås, I. Farup, and A. Rizzi, "Spatio-temporal retinex-inspired envelope with stochastic sampling: A framework for spatial color algorithms," Journal of Imaging Science and Technology 55(4), 40503-1 (2011).

18 G. Gianini, A. Manenti, and A. Rizzi, “Qbrix: a quantile-based approach to retinex,” JOSA A 31(12), 2663-2673 (2014). 
19 G. Gianini, A. Rizzi, and E. Damiani, "A retinex model based on absorbing markov chains," Information Sciences 327, 149-174 (2016).

20 G. Gianini, M. Lecca, and A. Rizzi, “A population-based approach to point-sampling spatial color algorithms," JOSA A 33(12), 2396-2413 (2016).

21 G. Gianini and A. Rizzi, "A fuzzy set approach to retinex spray sampling," Multimedia Tools and Applications , 1-26 (2017).

22 M. Lecca, A. Rizzi, and R. Serapioni, "Grass: A gradient-based random sampling scheme for milano retinex," IEEE Transactions on Image Processing 26(6), 2767-2780 (2017).

23 G. Sapiro and V. Caselles, "Histogram modification via differential equations," Jour. of Diff. Eq. 135, 238-266 (1997).

24 M. Bertalmío, V. Caselles, E. Provenzi, et al., "Perceptual color correction through variational techniques," IEEE Trans. on Image Processing 16, 1058-1072 (2007).

25 R. Palma-Amestoy, E. Provenzi, M. Bertalmío, et al., "A perceptually inspired variational framework for color enhancement," IEEE Transactions on Pattern Analysis and Machine Intelligence 31(3), 458-474 (2009).

26 M. Bertalmío, V. Caselles, and E. Provenzi, "Issues about the retinex theory and contrast enhancement," International Journal of Computer Vision 83, 101-119 (2009).

27 S. Ferradans, R. Palma-Amestoy, and E. Provenzi, "An algorithmic analysis of variational models for perceptual local contrast enhancement," Image Processing On Line 5(10), 219$233(2015)$.

28 G. Gronchi and E. Provenzi, "A variational model for context-driven effects in perception and cognition," Journal of Mathematical Psychology 77, 124-141 (2017). 
29 E. Land, “The Retinex theory of color vision," Scientific American 237, 108-128 (1977).

30 E. Provenzi and V. Caselles, "A wavelet perspective on variational perceptually-inspired color enhancement," International Journal of Computer Vision 106, 153-171 (January 2014).

31 T. J. Cooper and F. A. Baqai, "Analysis and extensions of the Frankle-McCann Retinex algorithm," Journal of Electronic Imaging 13, 85-92 (2004).

32 D. Marini and A. Rizzi, "A computational approach to color adaptation effects," Image and Vision Computing 18, 1005-1014 (2000).

33 G. Simone, G. Audino, I. Farup, et al., "Termite retinex: a new implementation based on a colony of intelligent agents," Journal of Electronic Imaging 23(1), 013006-013006 (2014).

34 E. Land, "An alternative technique for the computation of the designator in the retinex theory of color vision," in Proc. Nat. Acad. Sci. U.S.A., 83, 3078-3080 (1986).

35 D. Jobson, Z. Rahman, and G. Woodell, "Properties and performance of a center/surround Retinex," IEEE Transactions on image processing 6(3), 451-462 (1997).

36 B. Funt, F. Ciurea, and J. J. McCann, "Retinex in MATLAB," Journal of Electronic Imaging 13, 48-57 (2004).

37 D. Jobson, Z. Rahman, and G. Woodell, "A multiscale Retinex for bridging the gap between color images and the human observation of scenes," IEEE Transactions on image processing 6, 965-976 (1997).

38 R. Kimmel, M. Elad, D. Shaked, et al., "A variational framework for retinex," Intern. Jour. of Computer Vis. 52, 07-23 (2003).

39 J. Morel, A. Petro, and C. Sbert, “A pde formalization of retinex theory," IEEE Transactions on Image Processing 19(11), 2825-2837 (2010). 
40 E. Land, “The retinex," American Scientist 52(2), 247-264 (1964).

41 G. Simone and I. Farup, "Spatio-temporal retinex-like envelope with total variation," in Conference on Colour in Graphics, Imaging, and Vision, 2012(1), 176-181, Society for Imaging Science and Technology (2012).

42 M. Lecca, A. Rizzi, and G. Gianini, "Energy-driven path search for termite retinex," JOSA A 33(1), 31-39 (2016).

43 G. Simone, R. Cordone, R. Serapioni, et al., "On edge-aware path-based color spatial sampling for retinex: from termite retinex to light energy-driven termite retinex," Journal of Electronic Imaging 26(3), 031203-031203 (2017).

44 E. Provenzi, Computational Color Science: Variational Retinex-like Methods, John Wiley \& Sons (2017).

Edoardo Provenzi received his MS degrees in Theoretical Physics from Università di Milano, Italy, in 2000, and his PhD degree in Pure and Applied Mathematics from Università di Genova, Italy, in 2004. He was hired as Associate Professor of Applied Mathematics at Université Paris Descartes, France, in 2014 after ten years of post-doc positions in Italy and Spain. He obtained the position of full professor of Applied Mathematics at Université de Bordeaux, France, in 2017. His current research interests include image processing and mathematical models of human vision.

\section{List of Figures}

1 Digital (personal) reproduction of a typical Mondrian tableau used by Land and McCann for their color matching experiments. 
2 An example of (normalized) random spray. Normalization refers to the fact that the horizontal and vertical axes have been normalized between 0 and 1 . 